\title{
L'ARGENT DU RENOUVEAU: LES REVENUS DE LA ROYAUTÉ FRANÇAISE AU XII ${ }^{e}$ SIÈCLE
}

\author{
ERIC BOURNAZEL \\ Université de Paris-X Nanterre
}

(France)

\section{SOMMAIRE}

I. Des revenus encore traditionnels: A) Les structures domaniales. B) La consolidation du domaine.- II. Une meilleure utilisation des ressources: A) La pratique du fief rente. B) Des mentalités d'entreprise et de profit. C) Un souçi de contrôle et de gestion.

Un jour que Gautier Map, étudiant anglais et futur conseiller d'Henri Plantagenet, devisait avec le roi Louis VII, ils en vinrent à évoquer les richesses des différentes puissances du monde occidental: "L'empereur d'Allemagne -dit-il- à de bons hommes d'armes et des chevaux de guerre. Le roi d'Angleterre, il ne lui manque rien, les hommes et les chevaux, l'or et la soie, les pierres précieuses, les fruits, le gibier, il a tout". Et il termina en disant: "Nous en France, nous n'avons rien (nichil habemus) si ce n'est du pain, du vin et de la joie"1.

"Du pain, du vin et de la joie", l'expression résume assez bien l'entourage, la "compagnie", des rois capétiens au XII siècle.

A leur avenement, Louis VI et Louis VII ont en effet autour d'eux une bande de jeunes gens, de jeunes milites. Ce groupe constitue l'entoura-

'E. BOURNAZEL, Le gouvernement capétien au XIP siecle (1108-1180), Structures sociales et mutations institutionnelles, Paris, PUF, 1975, p. 125, note 26.

"Anuario de Eatudio Modiovales", 24 (1994) 
ge permanent du Capétien, sa familia, sa mesnie, sa maisonnée, sa suite qui l'accompagne dans ses déplacements et l'assiste dans ses opérations militaires ou ses choix politiques' ${ }^{2}$.

Pour la plupart, ce sont des cadets de familles issus des lignages chevaleresques des cites et des gros châteaux que le roi contrôle au coeur de son domaine. C'est un milieu modeste, descendant d'obscurs prévôts du $\mathrm{XI}^{\circ}$ siècle ou de gardiens de forteresses royales. Parfois, leur extraction est encore moins relevée et sur certains pèse même le soupçon de servitude ${ }^{3}$. Qu'importe d'ailleurs, leur dénuement est le gage le plus sâr de leur dévouement!

Les plus proches du roi ont reçu en sa maison des charges -sénéchal, chambrier, connetable, bouteiller, chancelier- dont les prérogatives ne sont guere définies mais qui rapportent a leurs titulaires les revenus des biens attachés à ces grands offices. Sous leurs ordres, des officiers inférieurs -chambellans, maréchaux, échansons, clercs ou notaires de la chapelle- s'emploient aux nécessités de la vie quotidienne: le pain et la viande, le vin et aussi le sommeil et la prière 4 .

C'est avec eux maintenant que le roi gouverne, rend la justice, expédie ses diplômés ou procède à des expéditions militaires. Les plus intimes, comme le chancelier Etienne de Garlande, son frère Anseau qui fut un temps sénéchal, ou encore l'abbé de Saint-Denis Suger, ami et historien des rois Louis VI et Louis VII, portent déjà le titre de conseiller. De fait, c'est de ces réunions informelles, et parfois débridées, autour du Capétien que sortira, dans la seconde moitié du XII ${ }^{\circ}$ siècle, le futur rouage essentiel de la monarchie: le conseils.

A leur place, à leur rang, les familiers du roi sont donc associés au premier chef à ce renouveau de l'autorité royale qui se dessine à notre époque, et dont ils ont été à bien des égards les principaux artisans.

\footnotetext{
${ }^{2}$ J.-P. Poly et E. Bournazel, La mutation féodale (XF-XIF siecles), Paris, PUF, 1991, $2^{\circ}$ ed., p. 277 s.; E. BOURNAZEL, Société féodale el fonction publique (fin $X^{*}$ - milieu XIIF sieccle) in Histoire de la fonction publique en France, Paris, NLF, I, 1993, p. 1428.

${ }^{3} \mathrm{E}$. BOURNAZEL, Le gouvernement capétien, op. cit., chap. III.

${ }^{4}$ Ibid, p. 94 s.

'Ibid, chap. IV; POLY et BoURNAZEL, La mutation féodale, op. cit., p. 284 s. ; E. BOURNAZEL, Les temps féodaux in J.-L. HAROUEL et alii, Histoire des institutions de l'époque franque de la Révolution, Paris, PUF, 1994, 6 ed., n $213 \mathrm{~s}$.
} 


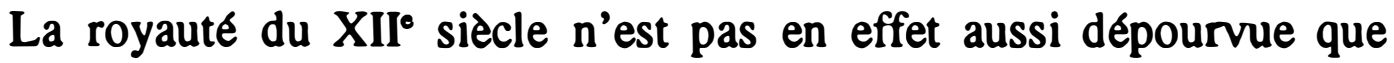
pourrait le laisser entendre la boutade de Louis VII. Avec Louis VI, le roi a commencé a remettre de l'ordre dans ses possessions au coeur de l'lle-deFrance, là où résidait l'essentiel de sa puissance. Préalable indispensable qui permettra à ses successeurs, Louis VII et Philippe Auguste, de se lancer dans des aventures extérieures et de s'imposer aux plus grands du royaume ${ }^{6}$

Mais le XII' siecle n'est pas seulement le temps du renouveau de la royauté, c'est aussi celui de la renaissance économique qui, amorcée au $\mathrm{XI}^{\circ}$, porte maintenant ses fruits. Cet essor est lié à un certain nombre de causes qui se combinent et s'enchaînent. Certains auteurs ont mis en avant l'esprit d'entreprise du seigneur, d'autres sa rapacité qui seraient aux origines d'une extension des surfaces cultivables, notamment par le jeu de défrichements, et d'une augmentation des rendements par la mise en oeuvre de certains progrès techniques.

De là, un accroissement et une amélioration sensibles des subsistances qui se seraient traduits par une notable poussée démographique, engendrant de nouveaux besoins qui, à leur tour, auraient entretenu et stimulé la dynamique économique. De là, aussi une intensification des échanges -liée peut-être également au recul des arabes en méditérannée- et une augmentation de la circulation monétaire, un essor du commerce et de l'artisanat que révele la montée en puissance des villes dont l'autonomie -sous forme de commune ou de consulat- commence à s'affirmer, face notamment aux puissances seigneuriales. Il en est de même, dans une certaine mesure, des communautés villageoises rurales?.

Les principales victimes de la nouvelle conjoncture sont bien souvent les petits sires et châtelains qui, faute d'avoir su s'adapter et sássurer des profits de leur seigneurie, sont maintenant absorbés par les principautés les plus importantes ou même par le roi ${ }^{8}$.

${ }^{6}$ E. Bournazel, Le gouvernement capétien, op. cit., p. 102 s.; Société féodale, op. cit., p. 265 8.; cf. aussi, Y. SASSIER, Louis VIl, Paris, Fayard, 1991 et J.W. BALDWIN, Philippe Auguste, Paris, Fayard 1991.

${ }^{7}$ Poly et Bournazel, La mutation féodale, op. cit., chap VIII; Ph. Contamane et alii, L'économie médiévale, Paris, A. Colin, 1993, chap. V; D. BARTHÉLÉMY, L'ordre seigneurial, Paris, Seuil, 1990, chap. 3.

'Sur tous ces thèmes, ef. par exemple, G. DUBY, La société aux Xle et XIle sizcles dans la région máconnaise, Paris, SEVPEN, 1971, p. $261 \mathrm{s.}$ 
L'argent du renouveau, ce n'est donc pas seulement celui du renouveau de la royauté proprement dite, c'est aussi celui plus général du renouveau économique. Dans une large mesure, le Capétien saura s'inspirer de ce mouvement et tirer profit de cet esisor. Et si, dans l'ensemble, ses revenus restent traditionnels; ils seront désormais mieux exploités, mieux utilisés.

\section{DES REVENUS ENCORE TRADITIONNELS}

Même s'il est le seul dans le royaume à être sacré, le Capétien, au $\mathrm{XII}^{\circ}$ siècle, apparaît encore, à bien des égards, comme un seigneur parmi les seigneurs. Comme eux, il a sa politique souvent réduite aux dimensions de ses possessions, au moins dans la première moitié du XII siecle; il exerce les mêmes prérogatives, perçoit les mêmes redevances et coutumes. Comme eux, il contrôle certains châteaux, certaines villes fortifiées et il a, en ses terres, ses clienteles de vassaux.

L'essentiel, la presque totalité des revenus ordinaires de la royauté est donc constitué par son domaine et c'est d'abord la pacification et l'extension de celui-ci, au cours du XII' siecle, qui contribueront à les accrô̂tre.

\section{A) Les structures domaniales}

Pour un juriste, le domaine royal, peut se définir comme l'ensemble des propriétés foncières (terres, châteaux, forêts) et aussi des droits et prérogatives (notamment de puissance publique) dont le roi jouit dans le royaume. Il comprend donc des éléments à la fois corporels (des biens, des immeubles) et incorporels (droits et prérogatives de toute nature) et ne se confond en aucun cas avec le royaume, même si, au fil du temps, avec l'affirmation de l'autorité monarchique, ils finiront par se rapprocher'.

Pour l'heure, on n'en est pas encore là: l'essentiel de ces possessions se concentre autour de Paris, au coeur de l'île-de-France; là où se situe, au XII ${ }^{\circ}$ siècle, le véritable théatre de la puissance royale, là d'où procèdent aussi les principales sources de revenus du Capétien. Ce domaine est

\footnotetext{
'E. Bournazel, Les temps féodaux, op. cit., p. 234.
} 
loin de constituer un tout homogene, il apparaît le plus souvent comme une sorte de mosaïque de propriétés et de droits, plus ou moins regroupés ou dispersés autour de certains lieux ${ }^{10}$.

Il est en effet des centres domaniaux de toute premières importances, c'est-à-dire des villes ou des villages dans lesquels le roi possède des domaines fonciers étendus, ainsi que des biens immobiliers. Par l'intermédiaire de ses prévôts et autres agents, il y perçoit de très nombreux droits, en argent ou en nature.

Ainsi à Paris, où il réside de plus en plus souvent, et qui deviendra sa capitale, le Capétien est propriétaire de terres, dont certaines plantées de vignes, d'un palais dans l'île de la Cité, et de plusieurs maisons. Il y possede en outre des celliers, des fours, des moulins et aussi une pecherie. Il y exerce le droit de monnaie et perçoit des revenus de justice, des droits de tonlieux, de péages, de sauf-conduit, de transit, des droits de foire et marchés. Il lève aussi un certain nombre de cens, particulierement sur les maisons et sur les métiers (ainsi ceux de boulangers ou changeurs). Meme si le roi ne possède pas de biens fonciers très étendus, ce sont là de nombreux biens immobiliers et surtout des droits dont les rapports sont très importants.

A Orléans, où siège aussi un prévôt royal, ses domaines fonciers ne sont pas non plus très vastes, puisqu'on est encore dans une ville. Le Capetien y possede toutefois, outre un verger et des terres, l'immense forêt voisine. Il y tient aussi des granges, des greniers, des celliers et y a un palais. Surtout, il perçoit les revenus de tonlieux péages, justice, sans doute de monnaie, et aussi des cens qui lui apportent des profits très appréciables. Il a enfin la possession d'un certain nombre d'églises paroissiales et de chapelles.

A Etampes (entre Orléans et Paris), au contraire, le roi est propriétaire de vastes domaines fonciers. Là encore, son prévôt lève en son nom les droits et les banalités habituelles. Il en est de même à Compiègne où il possede, en plus de son palais, d'immenses propriêtés foncières et aussi de vastes forêts qui lui rapportent des bénéfices non négligeables. Ici, Louis VII a abandonné la prévôté aux habitants moyennant de fortes redevances annuelles.

${ }^{10} \mathrm{~A}$. LUChaIRE, Louis Vl le Gros, annales de sa vie et de son règne (1081-1137), Paris, 1890, introd.; M. PACAUT, Louis VII et son royaume, Paris, SEVPEN, 1964, p. 127 8.,; Y. SASSIER, Louis VII, op. cit., p. $423 \mathrm{~s}$. 
Senlis est également un élément fondamental du domaine royal au $\mathrm{XII}^{e}$ siècle. Le roi y possede de vastes terroirs dont certains sont plantés de vignes et dont d'autres lui apportent sont des récoltes dont des taxes. Il y possede en outre le château, plusieurs moulins, des celliers, des granges et une pêcherie. Il y contrôle le transit, le tonlieu, la monnaie et le change; il en est de même à Poissy où là aussi, comme à Senlis, un prévôt administre ses biens et ses hommes, en son nom, et perçoit les redevances qui lui sont destinées.

A Laon, ville pourtant un peu à l'écart de la principale zone domaniale, le Capétien, en concurrence avec l'évêque, possede cependant des biens et des revenus importants sur lesquels veille son prévôt et le même phénomene se retrouve à Bourges, ville encore plus excentrée acquise par Philippe Ier, où le roi tire profit de vastes possessions.

En dehors de ces huit centres domaniaux principaux, il en est d'autres où le Capétien jouit de biens et de prérogatives, peut-être moins étendus, mais dont les revenus sont encore très appréciables. On les retrouve dans la vallée de la Seine, en amont de Paris, et dans les régions immédiatement voisines, autour de Corbeil, de Melun ou de Sens. Une autre zone de forte concentration se situe au sud de Paris, en direction d'Orleans et de la Loire, avec une intensité particulière au sud et au sud-est d'Etampes. Il en est de même en aval de Paris, dans la proche vallée de la Seine, aux portes de la Normandie, ainsi autour de Pontoise et de Mantes. Enfin, il est toute une série de biens et de droits, dispersés ici ou la, et qu'il serait vain d'énumérer, mais qui néanmoins rapportent, même lorsqu'il s'agit de possessions et de prérogatives purement domaniales ${ }^{11}$.

Au total, il s'agit là de sources de revenus considérables qu'il importe avant tout de protéger et de consolider.

\section{B) La consolidation du domaine}

La politique de Louis VI avait été de remettre de l'ordre entre ces différentes zones de possession, en ramenant à la raison, par la force, les sires et châtelains querelleurs. Le souci majeur de Louis VII sera plutôt de les accroître et de les consolider par des acquisitions nouvelles, le plus souvent situées à proximité d'anciennes possessions dont, au demeurant, elles

\footnotetext{
"M. PACAUT, op. cil.,; Y. SASSIER, op. cit.
} 
ne différaient pas fondamentalement dans leur nature, seigneuriale et domaniale.

Au début de son règne pourtant, du chef de son mariage avec Alienor, en 1137, Louis VII avait récupéré le duché d'Aquitaine. C'était là une acquisition d'envergure que son divorce en 1151 lui fera perdre. Ce n'est donc véritablement qu'à la fin du XII' siecle, au temps de Philippe Auguste que la royauté procédera à des accroissements notables et durables du domaine ${ }^{12}$.

Contrairement à leurs devanciers, les rois du $\mathrm{XII}^{\circ}$ siecle se sont montrés beaucoup plus soucieux de protéger ces domaines dont ils tiraient l'essentiel de leur puissance et de leurs revenus. Les concessions qu'ils peuvent consentir à l'Église ou à leurs fideles sont généralement de peu d'importance. Assez souvent, il s'agit de biens fonciers de faible superficie et donc de peu rapport ou encore de droits seigneuriaux ou domaniaux, de membres ici ou là en divers terroirs, ainsi des rentes assignées sur tel moulin ou tel pressoir ${ }^{13}$. Dans le même temps, nous le verrons, le Capétien s'efforce aussi de mieux gérer et contrôler ses possessions, intensifiant par la même ses revenus ${ }^{14}$.

Au demeurant, à la fin de son règne, Louis VII semble avoir été beaucoup plus riche qu'il ne le laissait entendre a Gautier Map, même s'il ne le sera jamais autant que son puissant vassal le roi d'Angleterre. Si l'on en croit, en effet, le témoignage, certes un peu tardif, en 1223, de Conon, prévôt de l'église de Lausanne: ses revenus seraient de l'ordre de 19.000 livres parisis par mois, soit plus de 220.000 livres par an, ce qui est déjà considérable $e^{15}$.

A cela il faut ajouter les revenus, plus épisodiques, mais très lucratifs que procurent les droits de régales et de dépouilles des évêchés vacants $^{16}$. Sans oublier non plus les revenus extraordinaires, c'est-à-dire ceux extérieurs au domaine et qui sont peu nombreux à notre époque. On peut citer, cependant, l'aide féodale perçue par Louis VII pour mener à

\footnotetext{
12J. W. BALDWIN, op. cit. ; J. KRYNEN, L'empire du roi. Idées et croyances politiques en France, XIlle-XVe siecle, Paris, Gallimard, 1993, p. 51 s.

${ }^{13}$ E. BOURNAZEL, Le gouvernement, op. cit., p. $65 \mathrm{s.}$

${ }^{14}$ Infra, Ile partie.

${ }^{15}$ M. PACAUT, Louis VII, op. cit., p. 159 8.; Y. SASSIER, Louis VII, op. cit., p .440.

${ }^{16} \mathrm{E}$. Bournazel, Les temps féodaux, op. cit., n 144 s.; M. PaCAUt, op. cit., p. $91 \mathrm{s.}$
} 
bien une croisade qui fut pourtant une opération politiquement et financièrement désastreuse ${ }^{17}$.

Dans la seconde partie de son règne, le roi saura parfaitement redresser la situation: sans doute parce que quelque chose avait changé, tant dans l'administration capétienne que dans les mentalités de ceux qui participaient au gouvernement royal.

\section{UNE MEILLEURE UTILISATION DES RESSOURCES}

Dans la gestion et dans l'administration de ses revenus, le roi, comme d'ailleurs son entourage, n'est pas resté étranger aux grands courants que portait au XII ${ }^{\circ}$ siecle le renouveau économique. De nouvelles pratiques financières font leur apparition, cependant que se développe un esprit dẻntreprise et de profit, lié à une volonté de contrôle et de gestion.

\section{A) La pratique du fief rente}

Au fil du temps, le Capétien se montre de plus en plus réticent à procéder à des amputations majeures de son domaine, même lorsqu'il s'agit de ses plus fideles serviteurs. Certes, les plus proches et plus influents d'entre eux ont bien reçu du roi, en fief, certaines seigneuries ou la garde de certains châteaux. Ils possèdent aussi des pressoirs, des fours, des moulins, mais rarement en nombre important. Ils exercent également certains droits ou prérogatives, sur les lambeaux de quelques terroirs dénombrés ${ }^{18}$. Mais, pour la plupart, leur richesse n'est pas foncière: matériellement, c'est d'abord de la maisonnée royale qu'ils dépendent. De fait, chaque année, les chevaliers de la maison du roi reçoivent à date fixe des vêtements et des cadeaux, et aussi une solde, stipendium, sous forme de fief rente.

Le fief-rente est une institution qui dérivait peut-être du vieux système de la provende, pratiquée par les grands établissements ecclésiastiques au profit de leur ministériaux et plus généralement des hommes de leur familia. Initialement distribuée tous les jours dans les réfectoires domesti-

\footnotetext{
${ }^{17}$ M. PACAUT, op. cit., p.46 8.; Y. SASSIER, op. cit., p. 1398.

${ }^{18}$ E. BOURNAZEL, Le gouvernement, op. cit., p. $83 \mathrm{~s}$.
} 
ques, la provende s'est souvent transformée en une rente d'abord en nature -du blé et du vin- et puis en argent, dans la seconde moitié du $\mathrm{Xl}^{\circ}$ siecle.

A notre époque, ces fiefs rentes versés par la royauté aux membres de son entourage -et dont la pratique coutumière est attestée dans le milieu du XII ${ }^{\circ}$ siecle- sont assurément en argent, comme d'ailleurs leur nom l'indique, beneficia denariorum. Il s'agit là de rentes, assignées le plus souvent sur les revenus de telle ou telle prévôté et qui étaient concédées en fief. Cette pratique est assurément très avantageuse pour le Capétien. Si d'aventure, le bénéficiaire vient à lui manquer, il est beaucoup plus aisé d'arrêter de verser l'argent -un seul ordre suffit- que de confisquer une terre ou de reprendre un château ${ }^{19}$.

De plus, ce système s'inscrit parfaitement dans le renouveau économique du temps, avec l'intensification des rentes seigneuriales et le développement de l'économie monétaire ${ }^{20}$.

\section{B) Des mentalités d'entreprise et de profit}

La volonté d'entreprendre et d'intensifier les profits, qui était au coeur même de la dynamique économique du XII ${ }^{e}$ siècle, se retrouve aussi bien au niveau des membres de l'entourage royal que de la royauté ellemême.

Quelle que soit la prudence du Capétien, un certain nombre de familiers se sont enrichis dans la proximité royale, et d'autant plus qu'ils n'hésitent pas à monnayer leur influence privilégiée auprès de leur maître. Ainsi, Etienne de Garlande, à la fois chancelier et sénéchal de Louis VI, et qui cumule les revenus de nombreuses dignités ecclésiastiques a amassé des biens considérables qui tranchaient avec la modestie de ses origines ${ }^{21}$.

Mais Etienne de Garlande ne se contente pas, comme certains, de profiter de l'essor économique qui emplit ses coffres. Il y participe également. Ayant reçu de l'église de Paris, à Rozay en Brie, un moulin et un four qui ne rapportaient que 3 muids et demi de farine et la même quantité de froment, il en augmente le rendement jusqu'à 8 muids dont, par contrat, il conserve le huitieme. A Epône, deux autres moulins procuraient 6 muids

\footnotetext{
${ }^{19} \mathrm{~J}$.-P. Poly et E. Bournazel, La musation féodale, op. cit., p. $281 \mathrm{~s}$.

${ }^{20} \mathrm{Ph}$. Contamine, L'économie médiévale, op., cit., p. 155 s. et $195 \mathrm{s.}$

${ }^{21}$ E. BOURNAZEL, Le gouvermeinent, op. cit., p. 35 s et 528.
} 
et demi, que se partageaient les chanoines et l'hospice des pauvres, Etienne, à qui ils les ont confiés, accroît les quantités de 9 muids et demi qu'il garde pour son propre usage. Dans la même localité, il double le rapport du pressoir et transforme en une redevance de 30 muids de vin les 40 sous que produisait un four. Enfin, il augmente très sensiblement le montant des taxes perçues sur les bateaux qui descendaient la Seine, chargés de vin ${ }^{22}$.

Sans doute, les quantités indiquées restent encore minimes mais l'esprit d'entreprise d'Etienne de Garlande ne devait pas s'exercer dans la seule poesté d'Epône. On peut citer aussi l'exemple de Suger qui s'illustra dans la bonne gestion des domaines de l'abbaye de Saint Denis ${ }^{23}$.

Non seulement les membres de la familia regis s'efforcent d'accumuler, ici ou là, des revenus, mais ils s'attachent aussi à les faire fructifier. Dans les villes renaissantes du XII ${ }^{\circ}$ siècle, se sont établis des marchands et des financiers. Très tôt, les chevaliers de l'entourage capétien sont en relation avec eux; ils ont mariés leurs filles à des changeurs italiens ou à des financiers lombards. Des liens étroits unissent désormais la mesnie royale au monde de l'argent. Certains familiers du roi, tel Henri le Lorrain au temps de Louis VI, sont d'ailleurs de véritables préteurs à gage dont les principaux clients sont les églises parisiennes. Quant au Clerc Cadurc, chapelain puis chancelier de Louis VII, abbé de Saint-Sulpice de Bourges et chanoine de Sainte-Croix d'Orleans, il est suffisamment riche pour faire des avances au roi, a la veille de la seconde croisade ${ }^{24}$.

La royauté, de son côté, n'est pas restée insensible à ce souci d'entreprise et de productivité, ne serait-ce que dans sa gestion domaniale. Dès l'époque de Louis VI, commence à s'amorcer une politique d'accroissement et de peuplement de l'espace cultivable du domaine, qui deviendra systématique dans les années 1150-1160, favorisée d'ailleurs par l'augmentation de la pression démographique ${ }^{25}$.

Pour attirer les hôtes, les défricheurs, le roi n'hésite pas à leur concéder des chartes de peuplement, à l'instar de celle de Lorris-en-Gâti-

\footnotetext{
${ }^{22}$ E. BOURNAZEL, Le gouvernement, op. cit., p. 59 s. ; M. BUR, Suger, Abbé de SaintDenis, Régent du royaume, Paris, Perrin, 1991, p. $132 \mathrm{~s}$.

${ }^{23}$ E. BourNaZEL, Suger and the Capetians, Abbot Suger and Saint-Denis, New-York, P., Gerson, 1986, p. 55 8.; M. BUR, Suger, op. cit., p. 172 s. et, SUGER, De administratione sua, trad M. BUR, Suger, La Geste de Louis VI, Paris, Imprimeric nationale, 1994, p. 219 s.

${ }^{24}$ E. BOURNAZEL, Le gouvernement, op. cit., p. 81 s. et 101.

${ }^{25} \mathrm{Ph}$. Contamene, L'économie médiévale, op., cit., p. $211 \mathrm{~s}$.
} 
nais. Les libertés qui y sont octroyées (protection contre les abus des prévôts et sergents royaux, dispenses de guet et d'ost, abolition de la plupart des corvées, diminution du cens des terres ou des maisons, exemption de taille et de redevances diverses sur les grains récoltés ou le vin produit, sauvegarde des marchands se rendant à la foire...) n'ont pas d'autre but que de favoriser le développement de ce type de bourgs ruraux, d'encourager l'essor de l'agriculture et du commerce, tout en plaçant la localité sous la domination du roi et de ses agents. Ce modele de charte sera assez largement diffusé dans le domaine royal dès la fin du XII ${ }^{\mathrm{e}}$ siècle $e^{26}$.

«L'octroi de chartes "globales" n'est pas le seul moyen utilisé par le Capétien pour fixer les populations et susciter un meilleur rendement des terres». Parfois, il se contente de renoncer seulement à certaines coutumes, parmi celles que les manants supportent le moins: ainsi en est-il des annulations de corvées, des renonciation au droit de gîte ou des exemptions de tailles et de services armés ${ }^{27}$.

Même si ces efforts témoignent d'une meilleure volonté d'organisation de la production, il est certain que les profits que le roi tire directement ou indirectement de l'activité des communautés rurales, ne sont pas toujours considérables, au regard notamment de ceux qu'il peut retirer des activités commerciales ou artisanales qui ont pour cadre les cités et les gros bourgs du domaine. Cèst de là, en effet, que proviennent désormais l'essentiel des revenus en argent. Aussi, le Capétien s'efforce de multiplier ou de réorganiser les foires et les marchés urbains. En multipliant, leur fréquence ou en augmentant leur durée, en accordant des sauf conduit aux marchands, des franchises ou des diminutions de tonlieux ou de taxes, il s'emploie à stimuler et à intensifier leur activité $e^{28}$.

Ici ou là également, le roi n'hésite pas à réglementer certains métiers, ainsi des boulangers a Pontoise ou des bouchers a Etampes. A Paris, Louis VII renouvelle en 1170 le privilège déja accordé aux marchands de l'eau par son pere Louis VI, cinquante ans plus tôt, et notamment le monopole de la navigation fluviale sur la Seine en amont de Mantes. De même,

\footnotetext{
${ }^{26}$ M. PACAUT, op. cit., p. 140 s.; Y. SASSIER, op. cit., p. 426 s.

${ }^{27}$ Y. SASSIER, op. cit., p. $430 \mathrm{~s}$.

${ }^{28}$ M. PACAUT, op. cit., p. $144 \mathrm{~s}$.
} 
il favorise et organise l'activité des changeurs établis sur le Grand-Pont a Paris $^{29}$.

Quant à la politique royale à l'égard du mouvement d'émancipation communale, elle est beaucoup plus ambiguë. Le Capétien n'hésite pas a favoriser et à encourager l'autonomie de certaines cités lorsqu'elles ne sont pas directement ou totalement placées sous son autorité seigneuriale. Dans le cas contraire, il se montre beaucoup plus réticent, mais il s'attache malgré tout à faciliter leur développement économique, source là encore de multiples profits et revenus ${ }^{30}$.

\section{C) Un souçi de contrôle et de gestion}

Dès la seconde moitié du XII ${ }^{e}$ siècle, en effet, la royauté se montre particulièrement soucieuse de remettre de lordre dans l'administration de son domaine comme dans la gestion de ses revenus.

Un signe d'ailleurs, ne trompe pas: le nombre des prévôts, qui sont les préposés par excellence à la gestion domaniale, passe de $20 / 25$, en 1137, à une bonne quarantaine à la fin du règne de Louis VII, en 1180, alors même que le domaine ne s'est pas considérablement accru en superfi$\mathrm{cie}^{31}$. Un tel phénomène témoigne de la volonté d'opérer un meilleur quadrillage administratif des villes et des campagnes en intensifiant l'action, et donc le rendement, de l'administration. Dès cette époque, la circonscription territoriale des prévôts commence à se préciser. Mais cela n'est pas toujours et partout réalisé. Dans bien des endroits, les limites de leur ressort d'intervention demeurent assez tloues, de là des chevauchements, des empiétements. Souvent aussi, les prévôtes sont très inégalement réparties: les unes entièrement urbaines, d'autres exclusivement rurales. Dans certaines villes, enfin, il y a parfois plusieurs prévôts dont l'un peut jouer un rôle prépondérant, ou qui administrent des ressorts bien séparés ${ }^{32}$.

Malgré ces incertitudes qui tendent cependant à s'estomper, la royauté s'efforce de reprendre en mains ses administrateurs et de mieux délimiter les pouvoirs de ses agents, afin d'en éviter les éventuels excès. Ainsi,

\footnotetext{
${ }^{29}$ Y. SASSIER, op. cit., p. 431-432.

${ }^{30}$ E. Bournazel, Société féodale, op. cit., p. $221 \mathrm{s.}$

${ }^{31}$ Y. SASSIER, op. cit., p. 423 s.; E. BOURNAZFL, Les temps féodaux, op. cit., $n^{\circ} 262$.

${ }^{32}$ E. BournazEl, Société féodale, op. cit., p. $290 \mathrm{~s}$.
} 
nombre de chartes et privilèges concédés aux communautés urbaines ou royales ont pour objet de restreindre ou d'énumérer, avec précision, les droits du prévôt. On lui enjoint de respecter les libertés et institutions municipales, à l'égard desquels on lui fait prêter parfois un serment de fídélité. Dans le même esprit, le capétien veille à ce que les charges ne deviennent pas héréditaires et se réserve à lui seul le soin d'y pourvoir. Mais l'innovation la plus importante reste dans doute l'instauration du système de la ferme qui se développe -peut-être à l'imitation de la Normandiedans la seconde moitié du XII ${ }^{e}$ siècle.

La prévôté était confiée a ferme, offert au plus offrant, généralement le temps d'un bail assez court qui n'excédait guère 3 ans. A Paris, par exemple, il s'agit d'un ferme annuelle tenue le plus souvent par de riches bourgeois qui administrent leur prévôté en gens d'affaire, mais très correctement semble-t-il, du moins au temps de Philippe Auguste. Aux termes fixés (Toussaint Chandeleur, Ascension) l'adjudicataire versait au Trésor royal la somme a laquelle il s'était engagé. La différence entre les recettes qu'il collectait et les dépenses qui lui incombaient constituait son bénefice ou ses pertes ${ }^{33}$.

Incontestablement, la ferme qui répondait à un souci d'organisation et de rentabilité financière présentait des avantages, ne serait-ce que de simplification et de clarification des comptes. Mais le systeme avait aussi l'inconvénient de pousser le prévôt, pour augmenter ou seulement assurer ses revenus, à se livrer à toutes sortes d'abus et d'exactions, parfois dénoncés par certaines communautés. D'où la nécessité de mieux contrôler encore ces agents et de ne pas hésiter à les révoquer lorsqu'ils commettaient certaines exactions. Dès 1173, le Capétien envoie d'ailleurs ses fideles conseillers en mission temporaire d'inspection pour le représenter, ici ou là, dans le domaine, et surveiller la gestion des prévôts et de leur agents. Nous sommes là aux origines des futurs baillis et sénéchaux ${ }^{34}$.

Incontestablement, dès la fin du règne de Louis VII, la perception des revenus royaux s'est assainie et régularisée. Leur volume, dans le même temps, s'est considérablement accru, intensifié, en liaison avec l'essor économique plus général, mais aussi du fait d'une meilleure administration. Ce n'est d'ailleurs pas un hasard si, à l'époque de Philippe Auguste,

${ }^{33}$ J. W. BALDWIN, Philippe Auguste, op. cit., p. 84 et p. $195 \mathrm{s.}$

${ }^{34}$ E. BouraAzel, Société féodale, op. cit., p. 293 8.; J.W. BALDWN, op. cit., p. 285 s. 
le Capétien a pu mettre en place un véritable budget et organiser les structures du Tresor ${ }^{35}$.

Plus généralement, cet argent a permis d'établir les rouages, les institutions, les agents, sur lesquels le roi, au XIII ${ }^{e}$ siècle, s'appuiera pour mettre en place et en oeuvre sa puissance souveraine retrouvee ${ }^{36}$. L'argent du renouveau économique a donc aussi, et peut-être surtout, servi le renouveau de la royauté.

\section{RÉSUMÉ}

Le XII ${ }^{\circ}$ siècle est à bien des égards, en Europe occidentale, en général, et dans le royaume de France en particulier, un période de renouveau économique. L'intensification des échanges, l'essor du commerce et la renaissance d'une économie monétaire profitent naturellement aux villes, aux princes les plus puissants et aussi à la royauté.

Si dans l'ensemble, les revenus du Capétien restent traditionnels, domaniaux, ils sont désormais mieux exploités, mieux gérés et mieux utilisés par un entourage royal, lui aussi renouvelé, dans ses hommes comme dans ses mentalités.

\section{SUMMARY}

In many respects, the XIIth century in Western Europe generally and more specifically the kingdom of France, is a time of economic revival. The intensification of trade, the expansion of commerce and the renaissance of a monetary economy are naturally profitable to towns, to the mightiest princes and also to the monarchy.

\footnotetext{
${ }^{33}$ J.W. BAIDWN, op. cit., p. $204 \mathrm{~s}$.

${ }^{36}$ E. BOURNAZEL, Les temps feodaux, op. cit., n $267 \mathrm{~s}$.
} 
Henceforth and considering human beings and attitudes of mind, the Capetian's income is better exploited, administered and used by royal entourage, which has also been renewed although this income remains, on the whole, traditional and domanial. 